\title{
Síndrome de Kartagener: diagnóstico neonatal. A propósito de un caso
} Kartagener syndrome: neonatal diagnosis. A case report

\author{
Dra. Ma. del Rocío Pérez Crespo a, Dra. Mercedes Fariñas Salto ${ }^{a}$ Dra. Rocío Chacón Aguilara, \\ Dra. Adriana Navas Carretero ${ }^{a}$, Dra. Eva Sanavia Morán ${ }^{a}$, Dra. Salomé Albi Rodríguez $z^{b}$ \\ Dra. Begoña Pérez-Moneo Agapito
}

\begin{abstract}
RESUMEN
El síndrome de Kartagener es una enfermedad hereditaria autosómica recesiva caracterizada por la asociación de discinesia ciliar primaria y la tríada situs inversus total, sinusitis crónicas y bronquiectasias. Su prevalencia varía en 1/15000-1/30000, pero se estima que muchos pacientes con discinesia ciliar primaria no han sido diagnosticados. Su presentación clínica es inespecífica y heterogénea, y no hay una única prueba gold standard para su diagnóstico. Esto, unido a las limitaciones y no disponibilidad de las pruebas, hace queel diagnóstico se retrase. Un diagnóstico y tratamiento adecuados de forma precoz modifican el pronóstico. En los últimos años, las sociedades han publicado algoritmos diagnósticos para pacientes con clínica sugestiva. Por ello, es importante una puesta al día y enfatizar en la necesidad de una sospecha clínica ante las manifestaciones clínicas de esta enfermedad.

Se presenta a un recién nacido con este síndrome diagnosticado por estudio genético en un hospital secundario.

Palabras clave: sindrome de Kartagener, discinesia ciliar primaria, situs inversus, sindrome de dificultad respiratoria del recién nacido.
\end{abstract}

\begin{abstract}
Kartagener Syndrome is an inherited autosomal recessive disorder characterized by primary ciliary dyskinesia and the triad of situs inversus viscerum, chronic sinus disease and bronchiectasis. Its prevalence varies from $1 / 15000$ to $1 / 30000$ but it is estimated that a lot of patients with primary ciliary dyskinesia have not been diagnosed as such. Its clinical presentation is non-specific and heterogeneous, and there is not a single, gold standard, diagnostic test. The diagnosis is often delayed because of these reasons and limitations and no availability of diagnostic tests. Early diagnosis and treatment change patient's prognosis. In addition, Scientific Societies have published recent diagnostic algorithm to evaluate the patient with suspected primary ciliary dyskinesia. Therefore,
\end{abstract}

a. Hospital Universitario Infanta Leonor, Madrid, España.

b. Hospital Universitario 12 de Octubre, España.

Correspondencia:

Dra. M. ${ }^{\text {a }}$ del Rocío Pérez Crespo: rociperez@hotmail.com

Financiamiento: Ninguno.

Conflicto de intereses: Ninguno que declarar.

Recibido: 30-7-2018

Aceptado: 3-1-2019 it is important to keep up to date with all the latest articles. We present the case of a newborn with this syndrome diagnosed by genetic analysis in a secondary care hospital.

Key words: Kartagener syndrome, primary ciliary dyskinesia, situs inversus, newborn respiratory distress syndrome.

http:/ / dx.doi.org/10.5546/ aap.2019.e292

Cómo citar: Pérez Crespo MR, Fariñas Salto M, Chacón Aguilar R, Navas Carretero A, et al. Síndrome de Kartagener: diagnóstico neonatal. A propósito de un caso. Arch Argent Pediatr 2019;117(3):e292-e296.

\section{INTRODUCCIÓN}

El síndrome de Kartagener es una enfermedad genética poco frecuente, definido por la tríada clínica característica situs inversus total, sinusitis crónicas y bronquiectasias. Fue descrito por primera vez por el neumólogo Manes Kartagener en $1933 .{ }^{1}$ Los pacientes con esta patología se incluyen entre los afectados por otro síndrome más amplio denominado discinesia ciliar rimaria (DCP). Esta se hereda con patrón autosómico recesivo y conlleva una alteración de la función y estructura del flagelo que afecta a todos los epitelios ciliados del organismo.,3

La prevalencia estimada es de 1/15000$1 / 30000$ personas, aunque trabajos recientes la sitúan en 1/10000 recién nacidos vivos. ${ }^{3}$ En 2006, se creó el primer grupo de trabajo internacional de la European Respiratory Society con el objetivo de mejorar la disponibilidad de los datos mediante un estudio de cohorte retrospectivo y la creación de un registro internacional prospectivo, ${ }^{4}$ lo que favoreció el conocimiento de esta patología y la elaboración de guías diagnósticas y terapéuticas. ${ }^{5,6}$

Actualmente, dado que, en el período neonatal, las bronquiectasias y sinusitis no están presentes y que un manejo adecuado y precoz mejora el pronóstico, este síndrome podría sospecharse por la presencia de DCP y situs inversus. ${ }^{2,7}$ 
Se describe un caso de síndrome de Kartagener que se manifestó precozmente con distrés respiratorio neonatal.

\section{CASO CLÍNICO}

Recién nacida a término (39 semanas) con peso al nacer de $2730 \mathrm{~g}$ (p11), primera hija de padre y madre sanos, no consanguíneos. Gestación controlada con analíticas y ecografías prenatales que no detectaban alteraciones significativas. Parto instrumental con fórceps, APGAR 9/10; no precisó reanimación al nacer. Sin antecedentes familiares de interés.

A las 41 horas de vida, ingresó desde la planta de obstetricia por el inicio de dificultad respiratoria. En la exploración física, presentaba tiraje, polipnea, regular estado general, mala coloración mucocutánea, y llamó la atención la auscultación del latido cardíaco desplazado a la derecha. Ante la sospecha de sepsis clínica, se extrajeron análisis de laboratorio, que constataron como dato de relevancia un aumento máximo de proteína $C$ reactiva (PCR) de $21 \mathrm{mg} / \mathrm{L}$, hemocultivos negativos, y se inició antibioterapia empírica con ampicilina y gentamicina. El citoquímico del líquido cefalorraquídeo era normal. En la radiografía de tórax realizada, se observaron corazón y fundus gástrico en el lado derecho e hígado en el izquierdo, con campos pulmonares sin signos de condensación ni escapes aéreos (Figura 1).

Figura 1. Radiografía de tórax-abdomen: situs inversus completo. Punta cardíaca a la derecha. Higado en el hipocondrio izquierdo y fundus gástrico en el hipocondrio derecho

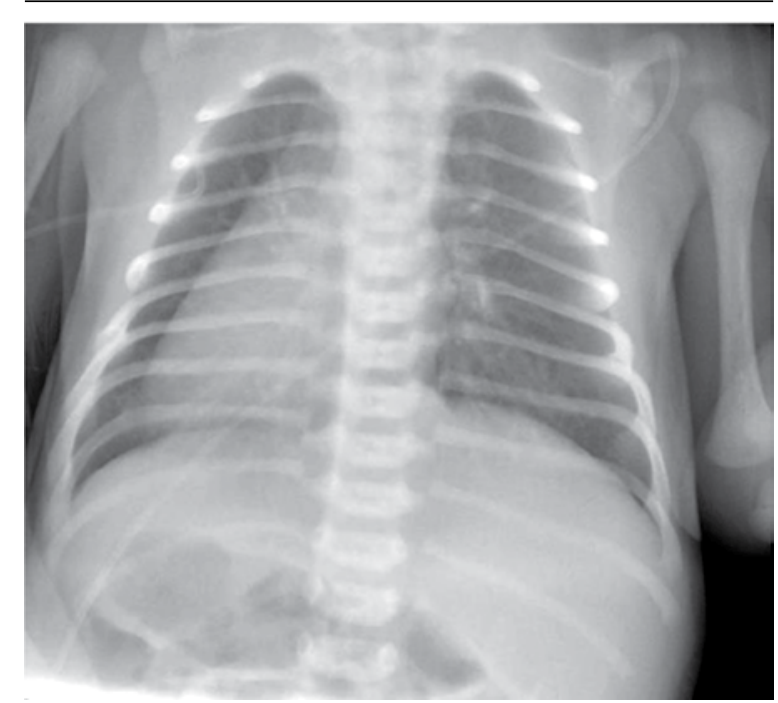

Como estudios complementarios, se realizaron ecografías transfontanelar y abdominal, en las que se confirmó el situs inversus total, sin otras malformaciones asociadas. Ecocardiograma: situs inversus, dextrocardia, dextroápex, corazón estructural y funcionalmente normal con signos indirectos de hipertensión pulmonar leve del recién nacido.

Precisó, desde su ingreso, soporte respiratorio, inicialmente, con presión positiva continua en las vías respiratorias (continuous positive airway pressure; CPAP, por sus siglas en inglés) nasal durante 2 días y, luego, con oxigenoterapia de alto flujo, flujo máximo de 5 latidos por minuto (lpm). Continuó con dificultad respiratoria moderada en los días sucesivos, a pesar de haber completado el tratamiento antibiótico, sin confirmación de infección por cultivos negativos, virológico de secreciones nasofaríngeas y controles de laboratorio normales. Ante la persistencia de la dificultad respiratoria inexplicable por otros motivos, unido a la presencia de situs inversus, se pensó en atribuir su causa al síndrome de Kartagener, por lo que se solicitó su estudio genético.

La evolución clínica posterior fue lenta pero favorable, sin necesidad de asociar otras medidas terapéuticas, lo que permitió la retirada de oxigenoterapia a los 20 días y el alta hospitalaria antes del mes de vida. De forma ambulatoria, se remitió para el seguimiento en consultas externas de Neumología y Fisioterapia Respiratoria. En el estudio genético, se detectaron dos variantes del gen CCDC39: c. $357+1 \mathrm{G}>C$ asociada a la DCP y c.1362G>A (p.Gln454Gln), que no había sido reportada previamente $\mathrm{y}$, por tanto, su significado clínico aún era desconocido. Ambas tenían una frecuencia muy baja en la población normal. Actualmente, la paciente tiene 5 meses de vida, presenta adecuada curva de ganancia pondoestatural y no ha presentado complicaciones ni requerimientos de oxigenoterapia asociados a su patología.

\section{DISCUSIÓN}

La DCP es la forma más frecuente de "ciliopatía", 8 en la que se ve afectada la motilidad de los cilios, lo que produce un aclaramiento mucociliar deficiente. ${ }^{9}$ Existe una gran variabilidad fenotípica debido a que los cilios móviles se encuentran, fundamentalmente, en la superficie apical del epitelio respiratorio, cilíndrico ciliado pseudoestratificado, que tapiza la vía aérea, pero también el oído medio, los 
senos paranasales, el epéndimo cerebral, las trompas de Falopio y los espermatozoides, entre otras localizaciones. Además, los cilios nodales embrionarios también son móviles y establecen la asimetría visceral y lateralidad del cuerpo; su ineficiencia da lugar a la aparición de situs inversus. ${ }^{8}$ El $50 \%$ de las DCP tienen un situs inversus total, que aparece tan solo en el 0,001\% de la población general, y el $25 \%$ de los pacientes con situs inversus total presentan una DCP. ${ }^{2}$

Las manifestaciones clínicas varían en función de la edad. En el período neonatal, más del $75 \%$ de los casos presenta dificultad respiratoria con necesidad de oxígeno sin causa aparente. En la infancia, es frecuente la tos crónica productiva, otitis medias, rinorrea y obstrucción bronquial recurrente tratados erróneamente como asma, pero con mala respuesta al tratamiento, mientras que, en los adolescentes y en los adultos, son más frecuentes las bronquiectasias, sinusitis, cefalea e infertilidad en los varones. ${ }^{2,3}$ En un estudio prospectivo publicado en 2016 por Leigh et al., se definieron cuatro características clínicas específicas en la infancia (dificultad respiratoria neonatal con necesidad de oxígeno inexplicable, tos húmeda, congestión nasal todo el año y alteraciones de lateralidad), que, solas o en combinación, eran predictivas de DCP, y presentaron una especificidad mayor del $96 \%$ con, al menos, 3 de estos síntomas. ${ }^{10}$

La clínica heterogénea e inespecífica favorece su diagnóstico tardío. Se publicó una encuesta internacional en la que el $35 \%$ de los pacientes había acudido a su médico más de 40 veces antes de ser remitidos para su diagnóstico. ${ }^{11} \mathrm{El}$ diagnóstico precoz podría disminuir los gastos de tratamiento, tal como se ha demostrado que ocurre en la fibrosis quística. ${ }^{12}$

En la actualidad, no existe una única prueba gold standard para el diagnóstico definitivo de DCP. Clásicamente, el diagnóstico se ha basado en la presencia de clínica sugestiva y demostración objetiva de las alteraciones estructurales del cilio con microscopía electrónica o disfunción ciliar mediante la evaluación de la frecuencia y el patrón de batido ciliar con video de alta resolución. Los inconvenientes son que solo se realizan en centros específicos, pueden existir cambios ciliares similares producidos por infecciones o contaminación y hasta el $30 \%$ de los pacientes presentan cilios con ultraestructura normal. ${ }^{2,5-7,13}$ A estos métodos diagnósticos se ha unido, en los últimos años, la genética que detecta los genes implicados en el $65 \%$ de los casos. Se han descrito más de 30 genes implicados; el $A N A H 5$ y $D N A l^{1}$ son los más frecuentes y se está intentando establecer una correlación genotipo-fenotipo. Pese a los avances, no necesariamente todas las mutaciones encontradas son patogénicas. ${ }^{3}$ Por ello, el estudio genético se usa para confirmar el diagnóstico en los pacientes con otras pruebas alteradas o alto índice de sospecha clínica. ${ }^{13}$ Otras pruebas diagnósticas son la inmunofluorescencia y la medición del óxido nítrico nasal. La precisión de esta última varía según el tipo de analizador y la edad del

TABla 1. Protocolo de seguimiento clínico y pruebas complementarias en pacientes con discinesia ciliar primaria recomendado por la Sociedad Española de Neumología Pediátrica

Seguimiento clínico

- Neumología: 2-4 veces / año en los niños y 1-2 veces/año en los adultos.

- Otorrinolaringología: 1-2 veces/año en los niños, cuando sea necesario en los adultos.

- Otros: fisioterapia respiratoria, nutricionista, medicina reproductiva, psicología.

Pruebas complementarias en el seguimiento

- Radiografía de tórax: cada 2-4 años.

- TAC de tórax: a los 5-7 años y cuando se requiera clínicamente según cada caso.

- Microbiología de secreciones respiratorias: 2-4 veces/ año.

- Cultivo de micobacterias atípicas: cada 2 años y si hay deterioro clínico sin causa aparente.

- Función pulmonar: 2-4 veces/año.

- Estudio de aspergilosis broncopulmonar alérgica: si hay sibilantes persistentes o deterioro clínico sin causa aparente.

Romero Rubio MT, Rovira Amigo S, Caballero Rabasco MA. Manejo del paciente afecto de discinesia ciliar primaria. Protoc diagn ter pediatr. 2017;1:423-437.

TAC: tomografía axial computada. 


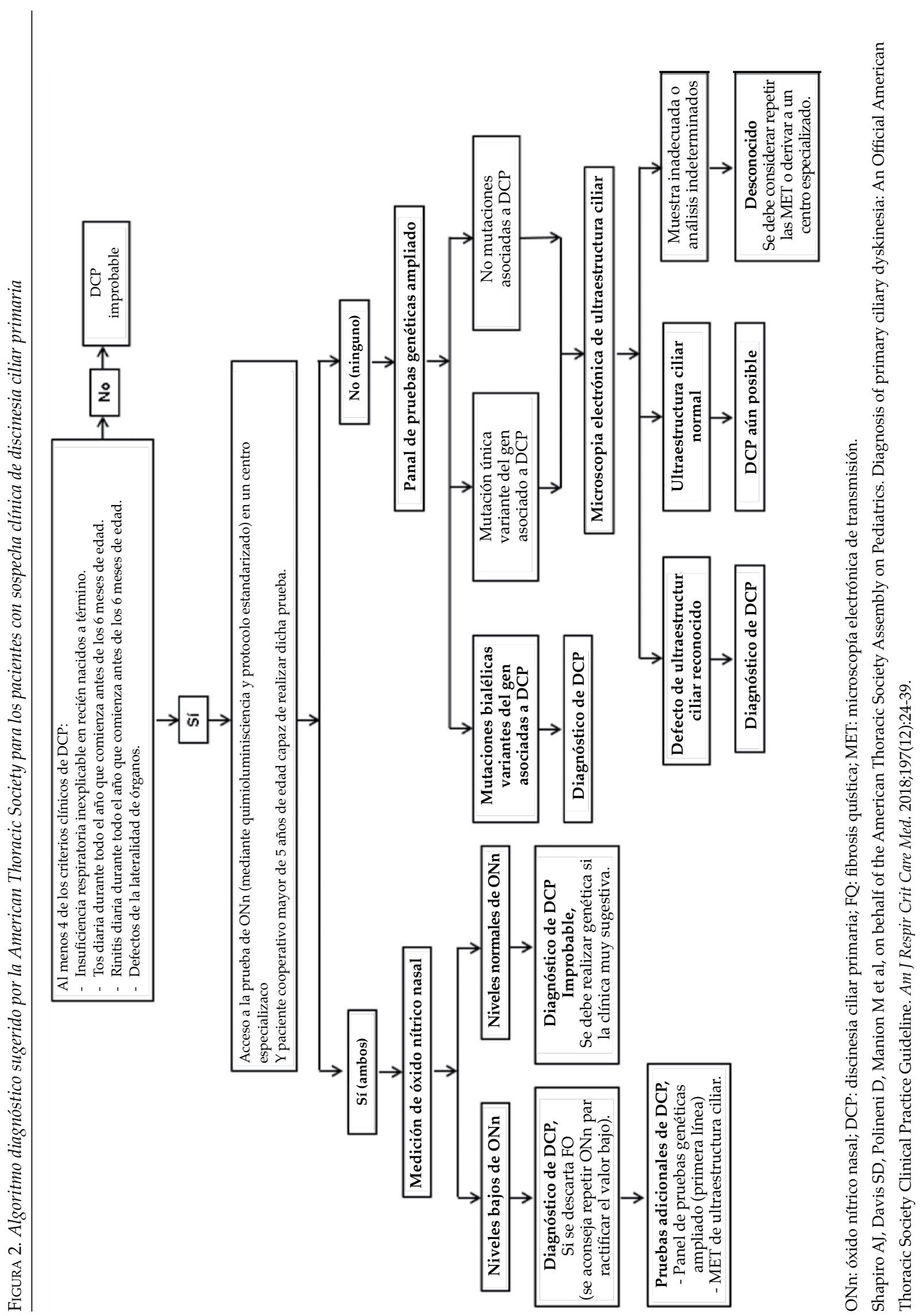


paciente (tasa de falsos positivos del $36 \%$ en los menores de 5 años), ${ }_{1}^{13}$ por lo que se recomienda usarla asociada a otras pruebas. Recientemente, la American Thoracic Society ha publicado un algoritmo diagnóstico para pacientes con clínica sugestiva de DCP (Figura 2). ${ }^{5}$ En el caso descrito, se solicitó el estudio genético, ya que existía un alto índice de sospecha, y otras pruebas, además de no estar disponibles, eran técnicamente difíciles de realizar por la edad de la paciente. El hallazgo de una mutación asociada a la DCP reforzó el diagnóstico de sospecha.

El manejo terapéutico actual no es específico; se basa en evidencias extrapoladas de la fibrosis quística, ya que, en su evolución, comparten la clínica de bronquiectasias y sinusopatía crónica. Los pilares del tratamiento son técnicas de aclaramiento mucociliar, fisioterapia respiratoria, ejercicio físico, prevención de infecciones con pautas de vacunación específicas, eliminación de exposición a desencadenantes inflamatorios e inhalaciones nebulizadas para diluir las secreciones, además del tratamiento específico de las complicaciones. ${ }^{2,7}$ En 2017, la Sociedad Española de Neumología Pediátrica publicó un protocolo de seguimiento de estos pacientes (Tabla 1). Cumpliendo el tratamiento y con un seguimiento estrecho, el pronóstico es, generalmente, favorable y llega, incluso, a tener una esperanza de vida normal. Pero, para ello, es fundamental un diagnóstico y tratamiento precoces, ya que su retraso se ha asociado a mayor número de sobreinfecciones y peor calidad de vida a largo plazo. En el futuro, el mejor conocimiento genético y fenotípico podría conducir a la creación de nuevas estrategias terapéuticas específicas. ${ }^{3,1}$

\section{REFERENCIAS}

1. Fajardo Dubon GE, Cárcamo Portillo GA, Rivera Rodríguez VM, Flores Raudales BL. Síndrome de Kartagener: Reporte de caso. Int J Med Surg Sci. 2017;4(2):1174-7.

2. Escribano Montaner A, Armengot Carceller M. Discinesia ciliar primaria. An Pediatr Contin. 2013;11(1):38-45.

3. Romero Rubio MT, Rovira AmigoS, Caballero Rabasco MA. Manejo del paciente afecto de discinesia ciliar primaria. Protoc Diagn Ter Pediatr. 2017;1:423-37.

4. Goutaki M, Maurer E, Halbeisen FS, Amirav I, et al. The international primary ciliary dyskinesia cohort (iPCD Cohort): methods and first results. Eur Respir J. 2017;49(1):1601181.

5. Shapiro AJ, Davis SD, Polineni D, Manion M, Rosenfeld M, et al. Diagnosis of Primary Ciliary Dyskinesia: An Official American Thoracic Society Clinical Practice Guideline. Am I Respir Crit Care Med. 2018;197(12):e24-39.

6. Lucas JS, Barbato A, Collins SA, Goutaki M, et al. European Respiratory Society guidelines for the diagnosis of primary ciliary dyskinesia. Eur Respir J. 2017;49:1601090.

7. Mirra V, Werner C, Santamaria F. Primary Ciliary Dyskinesia: An Update on Clinical Aspects, Genetics, Diagnosis, and Future Treatment Strategies. Front Pediatr. 2017;5:135.

8. Penín M, López Neyra A, Albi S, Alonso E, Gimeno A. Actualización en discinesia ciliar primaria: manejo actual y perspectivas de futuro. Rev Patol Respir. 2018;21(1):17-24.

9. Rubbo B, Lucas JS. Clinical care for primary ciliary dyskinesia: current challenges and future directions. Eur Respir Rev. 2017;26(145):170023.

10. Leigh MW, Ferkol TW, Davis SD, Lee HS, et al. Clinical Features and Associated Likelihood of Primary Ciliary Dyskinesia in Children and Adolescents. Ann Am Thorac Soc. 2016;13(8):1305-13.

11. Behan L, Dunn Galvin A, Rubbo B, Masefield S, et al. Diagnosing primary ciliary dyskinesia: an international patient perspective. Eur Respir J. 2016;48(4):1096-107.

12. KuehniCE, Frischer T, Strippoli MPF, Maurer E, et al. Factors influencing age at diagnosis of primary ciliary dyskinesia in European children. Eur Respir J. 2010;36(6):1248-58.

13. Kuehni CE, Lucas JS. Diagnosis of primary ciliary dyskinesia: summary of the ERS Task Force report. Breathe (Sheff). 2017;13(3):166-78.

14. Behan L, Rubbo B, Lucas JS, Dunn Galvin A. The patient's experience of primary ciliary dyskinesia: a systematic review. Qual Life Res. 2017;26(9):2265-85. 